УДК 612.424:613.693:615.471

\title{
НОВЫЙ КЛАСС ЛЕЧЕБНО-ДИАГНОСТИЧЕСКИХ КОМПЛЕКСОВ НА ОСНОВЕ СПЕКТРАЛЬНОГО АНАЛИЗА БИОАКУСТИЧЕСКОЙ АКТИВНОСТИ ГОЛОВНОГО МОЗГА ЧЕЛОВЕКА
}

\author{
Пегова Е. В., Меркулова Г. А., Лебедев Ю. А., Шабанов Г. А., Рыбченко А. А. \\ ФГБУН Научно-исследовательский центр «Арктика» ДВО РАН, г. Владивосток \\ E-mail: neurokib@mail.ru
}

\begin{abstract}
Обсуждается разработка инструментальных методов диагностики функциональных состояний организма для экстремальных природных зон обитания человека. Предложен новый диагностический аппарат для регистрации спектра биоакустической активности головного мозга «РС АЭГ01». Спектр отснятых сигналов вибраций головы в диапазоне частот от 0.13 до 27 Гц представлен в виде многочастотной матрицы множества функциональных состояний висцерального анализатора - «висцерома». Количество спектральных гармоник - 8400. Для автоматической оценки полученного рельефа активации множества вегетативных функций разработан ряд интегральных показателей: вегетативный индекс, индекс напряжения, индекс индивидуального здоровья, индекс децентрализации. На примере регистрации вегетативного индекса описано проведение функциональных проб: степ-теста, теста с возвратным дыханием. Аппарат позволяет оценивать функциональное состояние и адаптационные возможности организма, функционально-топическую диагностику заболеваний, неинвазивный скрининг ранних форм социально значимых заболеваний, включая онкологические. Обсуждаемая технология разрешает создать сеть полностью автоматизированных диагностических пунктов мониторинга индивидуального здоровья населения, актуальных для сложных условий эксплуатации Севера и Северо-Востока России, которые будут замыкаться по каналам связи на центральный сервер обработки информации.
\end{abstract}

Ключевые слова: акустоэнцефалограмма, регистрация спектра, неинвазивный скрининг, функциональное состояние, многочастотная матрица, рельеф активации висцеральных функций.

DOI: $10.34078 / 1814-0998-2020-2-114-122$

\section{ВВЕДЕНИЕ}

Экстремальная зона обитания человека на Севере и Северо-Востоке России предъявляет к организму значительные требования, вынуждая его использовать дополнительные социальные и биологические средства защиты от неблагоприятного воздействия внешней среды. Адаптация в этих условиях достигается путем напряжения и сложной перестройки гомеостатических систем организма. Правильная диагностика патологических состояний человека в этих условиях всегда будет связана с учетом и верной оценкой фазы адаптивного процесса и преморбидного фона для каждого конкретного индивидуума.

Современные подходы к неинвазивной оценке функционального состояния организма базируются в основном на изучении функций нервной системы. Относительная устойчивость состояния

(C) Пегова Е. В., Меркулова Г. А., Лебедев Ю. А., Шабанов Г. А., Рыбченко А. А., 2020 и деятельности нервной системы, разнообразные формы проявления ее неустойчивости свидетельствуют о различных, в том числе патологических, сдвигах функционального состояния организма (Зимкина, 1972). Ритм сердца находится в тесной и постоянной взаимосвязи с центральной и вегетативной нервной системой, является важнейшим индикатором ее состояния. Изменения сердечного ритма и показателей центрального кровотока важное звено в адаптации организма к условиям внешней среды (Баевский, 1979, 1997; Conny, 1993). В настоящее время математический анализ сердечного ритма широко применяется в физиологии спорта и медицине как один из наиболее информативных методов оценки состояния организма (Булатецкий, 2002). Другой, часто используемый показатель состояния нервной системы - уровень общей неспецифической реактивности организма человека, регистрируемый посредством определения болевой чувствительности (Мулик и др., 2009). Это генетически обусловленный интегральный критерий, 
отражающий степень общей чувствительности и реактивности организма к различным экзогенным воздействиям. Регистрация непосредственно сигналов с головного мозга - современная ЭЭГ, считается наиболее пригодным методом выявления мозговых функций и очень чувствительным инструментом определения церебральных патологий. Преобладание или недостаток активности различных ЭЭГ частотных полос у людей ассоциируется с различными функциональными состояниями мозга, что определяет взвешенный вклад метода в оценку состояния различных функций и организма в целом. Такой подход позволил характеризовать с помощью ритмических ЭЭГ-паттернов широкий спектр функциональных состояний организма (Жирмунская,1995; Кирой, 1998; Данилова, Крылова, 2002; Гнездицкий, 2004). Однако реализованных в практику систем по оценке функционального состояния организма человека с помощью съема и анализа ЭЭГ разработано не было.

В настоящее время актуальна разработка объективных неинвазивных инструментальных методов диагностики, индексных критериев адаптации, интегральных показателей различных функций организма и его систем. В современных условиях роста заболеваемости важным принципом является возможность скрининга ранних форм различных патологических состояний, включая онкологические заболевания. При этом, в связи со сложными климатическими условиями, частым отсутствием необходимых специалистов, дополнительно важными требованиями к медицинской технологии остаются автономность, простота в эксплуатации, доступность, неинвазивность, скорость и возможность передачи таких индексных показателей по каналам связи.

В нашей лаборатории разработан принципиально новый подход в изучении ритмической активности головного мозга с помощью спектрального анализа высокого разрешения акустоэнцефалограммы (АЭГ) - суммарных механических вибраций, которые распространяются от нейронных сетей, внутримозговых сосудов, мышечной системы головы и тела человека. Диагностический аппарат за 3 мин съема получает спектральную многочастотную матрицу, анализ которой может дать полную информацию о состоянии вегетативной сферы организма, выделить системные и локальные диагностические признаки для оценки адаптабельности, дисфункций и патологических состояний (Шабанов и др., 2005, 2017).

Цель исследований: оптимизация ранней оценки уровней здоровья, дизрегуляционных расстройств, развития новообразований благодаря использованию в алгоритме функциональнотопической диагностики индексных показателей, полученных с помощью диагностического аппарата «Регистратор спектра акустоэнцефалограммы (РС АЭГ-01)».

\section{ОБЪЕКТ И МЕТОДЫ ИССЛЕДОВАНИЯ}

Дизайн настоящего исследования носил «учебный» характер. Предусматривалось получить предварительные результаты и проверить выдвинутые гипотезы для планирования и последующих широкомасштабных исследований. Исследовали в основном практически здоровых добровольцев - 26 мужчин в возрасте от 28 до 60 лет. Критерием включения служил профилактический осмотр без признаков обострения хронических заболеваний. Критерием исключения наличие в анамнезе неврологических заболеваний.

Для регистрации спектра акустических сигналов головы был разработан опытный образец медицинского аппарата под названием «Регистратор спектра акустоэнцефалограммы РС АЭГ01». Аппарат посредством датчиков вибраций, которые распространяются от нейронных сетей, внутримозговых сосудов, мышечной системы головы и тела человека, регистрировал суммарную ритмическую активность отдельно для правого и левого полушария и выполнял ее спектральный анализ высокого разрешения.

В качестве индукционных датчиков вибраций использовали две дифференциальные пары катушек, которые находились в постоянном магнитном поле Земли около 40-50 мкТл. Активные катушки (правая и левая) располагались в височнотеменных областях левого и правого полушария. Для защиты от разнообразных помех дифференциальные пассивные катушки были вынесены за пределы головы на расстояние не менее 1 м и размещены строго в плоскостях ориентации активных катушек. Такая схема расположения индукционных датчиков позволяла надежно снимать суммарную вибрационную активность левого и правого полушария головы в диапазоне частот от 27.0 до 0.13 Гц. Расположение датчиков на голове человека показано на рис 1. При вибрации головы индукционные датчики, находясь в естественном постоянном магнитном поле Земли, генерируют электрический сигнал, пропорциональный скорости вибраций. Параметры катушки - 8000 витков, диаметр 70 мм. Средняя амплитуда сигнала на выходе индукционного датчика 20 мкВ.

С акустических датчиков электрические сигналы поступали на входы двухканального усилителя. Цифровая фильтрация и спектральный анализ при быстром Фурье-преобразовании имели свои особенности. Весь диапазон изучаемого сигнала от 0.1 до 27 Гц был разбит на 4200 полос, центральные частоты которых образуют геометрическую прогрессию с q $=2^{1 / 24}$ с опорной частотой 


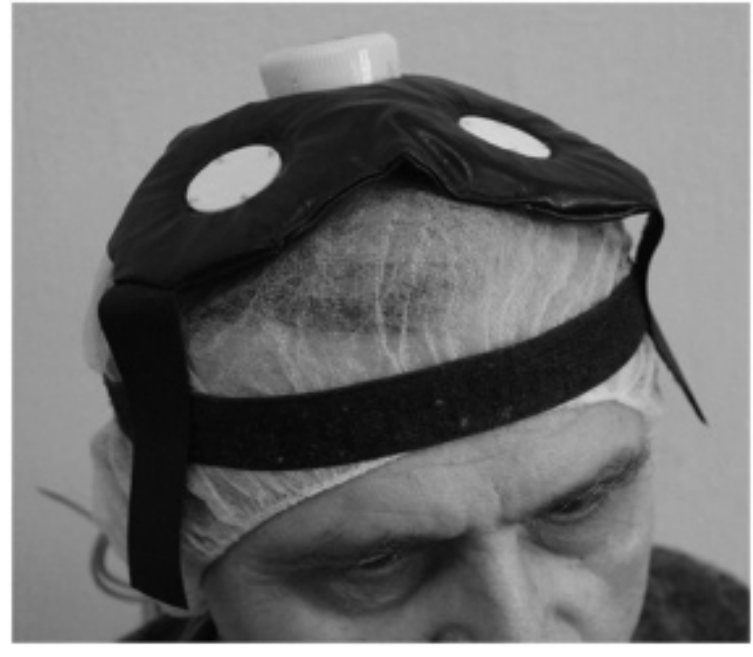

Рuc. 1. Расположение индукционных датчиков вибраций головы для съема акустоэнцефалограммы (АЭГ)

Fig. 1. Location of induction sensors of the head vibration for registering acoustic encephalogram (AEG)

27.005 Гц, при этом спектральные гармоники, попавшие в одну из 4200 полос, интегрировались по амплитуде. Получившиеся с левого и правого полушария 8400 центральных частот с соответствующими амплитудами спектральной оценки сворачивались в две спектральные матрицы размером $24 \times 175$ частотных ячеек. Время суммации сигнала по каждой спектральной ячейке выбрано 160 с. Такое время соответствует характеристикам исследуемой пластичности активирующей системы мозга как среднее время смены функциональных состояний при мозговой деятельности. Частота квантования сигнала 256 Гц (Патент № 18056, 2018). Подобный подход к спектральному анализу был осуществлен при изучении функциональной активности гиппокампа в работе И. Я. Подольского с соавторами (2000), модернизирован нами для частотного анализа ретикулярных структур активирующей системы мозга и получения спектральной матрицы «множества функциональных состояний». Выбор длительности записи информационного кадра 160 с позволил получить надежную информацию о спектре длительнотекущих процессов головного мозга, таких как реакции висцерального анализатора при различных функциональных пробах, эмоциональные реакции, реакции вкусового и обонятельного анализаторов и т. п. Спектральная матрица «множества функциональных состояний» отражает непрерывную «фоновую» активность центральной нервной системы по поддержанию функциональных состояний отдельных периферических эффекторов. Высокая разрешающая способность до 8400 частотных полос в матрице, специфичность к различным рефлекторным группам позволили назвать такую регуляторно- анализаторную волновую структуру «висцером» (Шабанов и др., 2011).

При изучении генеза АЭГ в ряде наших исследований осуществляли синхронную запись АЭГ и ЭЭГ (Шабанов и др., 2017), где была показана значимая корреляция сигналов для различных частотных полос. Чтобы верифицировать ряд индексов оценки состояния человека, использовали синхронную запись АЭГ и кардиоинтервалограммы (КИГ). Был использован комплекс для обработки кардиоинтервалограмм и анализа вариабельности сердечного ритма «Варикард 2.51» (Семенов, 2010a). Время записи кардиоинтервалограммы - 5 мин.

Статистическую обработку результатов исследования выполняли на персональном компьютере с помощью приложения Microsoft Excel. При расчетах использовали пакет прикладных программ «Статистика 6.0». Из числа основных характеристик относительной статистики для каждого вариационного ряда полученных результатов исследования вычисляли средние арифметические величины (М), средние квадратичные отклонения $(\sigma)$, относительные величины $(\mathrm{P})$, корреляцию между парой функций (r). При сравнительном анализе групп применяли t-критерий Стьюдента, коэффициент парной ранговой корреляции Спирмена (r), достоверности коэффициента корреляции. Нулевая гипотеза о различиях между признаками анализируемых выборок принималась при $\mathrm{p} \leq 0.05$.

\section{РЕЗУЛЬТАТЫ ИССЛЕДОВАНИЯ И ОБСУЖДЕНИЕ}

Исследование связи снижения адаптационных возможностей организма с заболеваемостью при массовых осмотрах населения показало, что длительное выраженное напряжение регуляторных механизмов ведет к невосполнимому расходованию функциональных резервов и, в итоге, - к развитию патологических состояний или к нарушению сложившихся физиологических компенсаций. Напряжение регуляторных систем - это неспецифический ответ организма на любые неблагоприятные для него воздействия, требующие мобилизации функциональных резервов. Определенное напряжение механизмов регуляции зависит от возраста, пола, индивидуальных особенностей, уровня здоровья, но не выходит за рамки так называемой физиологической нормы. В тех случаях, когда окружающие условия среды требуют от организма повышенных усилий и напряжение регуляторных систем выше обычного, «диагностируют» функциональное напряжение - умеренно, значительно или резко выраженное. Донозологические состояния являются пограничными между нормой и патологией. Они предшествуют развитию болезни и отража- 
ют снижение адаптационных возможностей организма (Баевский, 1979, 1997; Семенов, 2010б).

При анализе спектральной матрицы «висцером» с использованием аппарата «РС АЭГ-01» был разработан ряд интегральных показателей индексов, которые позволяли определять напряжение регуляторных систем организма. Среди них «вегетативный индекс» (ВИ), отражающий отношение эрготропных и трофотропных систем организма; «стресс-индекс» (СИ), отражающий активность симпатической системы организма; индекс индивидуального здоровья (ИИЗ), позволяющий оценить расположение пациента на шкале здоровья от сбалансированности функций до стадии субкомпенсации и ограничения функциональных возможностей организма. Для выделения группы риска развития злокачественных опухолей был разработан «индекс децентрализации» (ИДЦ).

Как пример, приведем несколько функциональных проб с использованием вегетативного индекса ВИ, который характеризует баланс эрготропных и трофотропных центральных влияний, контролирующих преимущественно адренергические и холинергические исполнительные механизмы. Индекс изменяется от 0 до 20 ед.: 1-3 ед. нормотония; выше 3 ед. - симпатикотония; ниже 1 ед. - ваготония. Аналогом ВИ при анализе кардиоинтервалограмм являлся стресс-индекс SI.

У практически здорового испытуемого при воздействии физической нагрузки (степ-тест) усиливается активность симпатического отдела $\mathrm{BHC}$, что влечет за собой повышение индексов ВИ и SI (состояние симпатикотонии). Для ВИ такое значение должно быть выше 2.5 ед., для SI - выше 150 ед. В исследовании принимали участие 23 практически здоровых добровольца в возрасте 2026 лет, не имеющих жалоб на здоровье. Осуществлялась синхронная запись АЭГ и кардиоинтервалограммы (Семенов, 2010а). Запись одного съема АЭГ длилась 160 с.

На первом этапе проводили три фоновых съема с интервалом 3-5 мин. Доброволец находился в физиологических условиях полного покоя с закрытыми глазами в положении сидя. Далее съем информации прекращали. Проводили функциональную пробу с физической нагрузкой (стептест). Доброволец выполнял подряд 120 подъемов на ступеньку высотой 0.5 м. После прекращения нагрузки проводили 5 съемов периода восста- новления с интервалом 3-5 мин, в полном покое с закрытыми глазами в положении сидя.

После 30-минутного отдыха, во второй части эксперимента проводили гипоксическогиперкапническую функциональную пробу (пробу с возвратным дыханием). Добровольцу также делали три фоновых съема в положении сидя. Далее предлагали дышать в мешок объемом 20 л в течение 3 мин. Во время этой процедуры шла одновременная запись АЭГ и кардиоинтервалограммы. Для удобства и более точной записи мешок должен был держать ассистент. Следующим этапом был съем информации без мешка этапа восстановления - один съем в полном покое, спокойное дыхание (рис. 2-4).

Реакция ВИ на функциональные пробы основной показатель адаптабельности организма к внешней среде, степени его тренированности, способности переносить физическую нагрузку. Критерии для анализа таких функциональных проб отработаны и опубликованы во многих работах (Баевский, 1979, 1997; Семенов, 2010б). Здесь мы отметим высокую корреляцию поведения во времени индексов ВИ и $\mathrm{Si}$ при синхронных съемах АЭГ и КИГ. Для 23 добровольцев средняя парная корреляция для степ-теста ока-

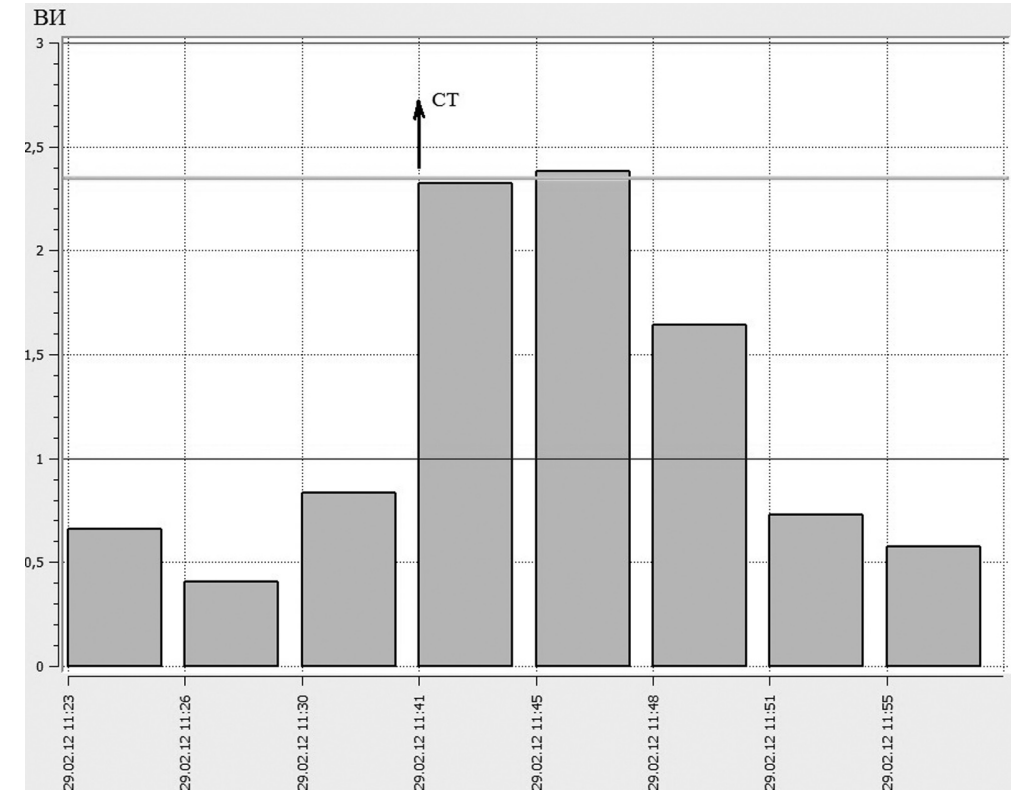

Puc. 2. Пример реакции вегетативного индекса ВИ на степ-тест у испытуемого А. По оси абсцисс - ВИ в относительных единицах. По оси ординат - столбчатая диаграмма значений ВИ во времени. Первые три съема - фон. СТ - начало восстановительного периода после степ-теста

Fig. 2. Example of the response of autonomic index AI (ВИ) on the step test of Subject A. On the abscissa axis, the AI is in relative units. The bar graph of the values of the AI in time is located on the ordinate axis. The first three registering sessions were considered background. ST (CT) is the beginning of the recovery period after the step test 

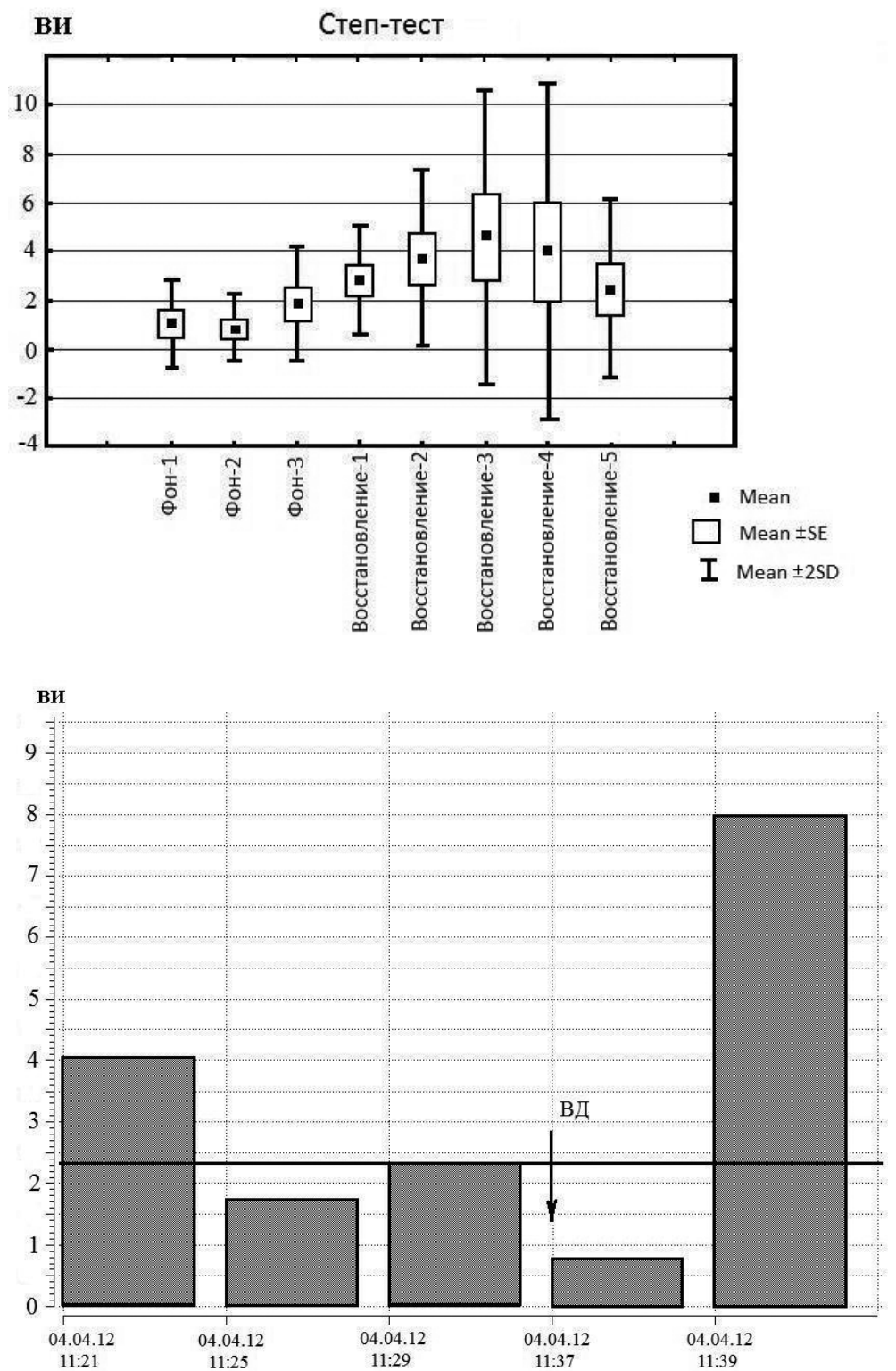

Рuc. 3. Статистический анализ реакции ВИ на степ-тест у 23 практически здоровых испытуемых. По оси ординат - вегетативный индекс ВИ. По оси абсцисс - реакция ВИ во времени. Mean - среднее значение. SD - стандартное отклонение; SE - стандартная ошибка при $\mathrm{p} \leq 0.05$

Fig. 3. Statistical analysis of the AI (BИ) response to a step test in 23 practically healthy subjects. The abscissa axis is Autonomic Index AI. The ordinate axis is the AI response in time. Mean stands for the average value; $\mathrm{SD}$, for the standard deviation; $\mathrm{SE}$, for the standard error $\mathrm{p} \leq 0.05$

Puc. 4. Пример реакции вегетативного индекса ВИ на функциональную пробу с возвратным дыханием у испытуемого А. По оси абсцисс ВИ в относительных единицах. По оси ординат расположена столбчатая диаграмма значений ВИ во времени. Первые три съема - фон. ВД - съем во время дыхания в мешок и далее, начало восстановительного периода

Fig. 4. Example of the autonomic index $\mathrm{AI}(\mathrm{B}$ ) $)$ response to a functional re-breathing test in Subject A. On the abscissa axis, the AI is in relative units. The bar graph of the AI values in time is on the ordinate axis. The first three registering sessions were considered background. Re-breathing, RB (ВД) is registered during breathing in the bag, and further on, the beginning of the recovery period залась 0.87; корреляция для теста с возвратным дыханием - 0.78. Это говорит о возможности использования регистрации акустоэнцефалограммы АЭГ для проведения подобных функциональных исследований.

Для скрининга ранних форм эпителиальной раковой опухоли у пациента был разработан индекс децентрализации ИДЦ (Патент № 2661098 , 2018).

Было установлено, что снижение адаптационного трофического потенциала нервной системы (понижение амплитудного значения и подвижности спектральных составляющих функции активности рецепторов) напрямую связано с децентрализацией участка органа и нарастающей нервной дистрофией. Если децентрализация наблюдается длительное время, то в органе фиксировалось нарастание неконтролируемой клеточной пролиферации, степень которой напрямую связана с выраженностью де- централизации и с количеством вовлеченных в этот процесс рефлекторных звеньев (клеточная гиперплазия, метаплазия, дисплазия). Финалом застойной децентрализации ткани или органа в большинстве случаев являлось развитие ацидоза, закисление среды, переход клеток на анаэробный тип питания и, как правило, развитие злокачественной опухоли (Хамошин, 1998; Юдин, 1999; Рольщиков, 2002; Шабанов и др., 2011).

Проведение исследований на аппарате «РС АЭГ-01» показало, что каждая спектральная ячейка матрицы, при приближении ее к нулевым амплитудным значениям, полном снижении подвижности (дисперсии параметра амплитудной оценки), оказывает влияние на периферические органы, равносильно денервации или децентрализации. Были разработаны амплитудные критерии для нормальной ткани, частичной и полной децентрализации. Для удобства 
расчетов был разработан индекс децентрализации ИДЦ, которой изменялся от 0-30 в норме до 1500-2000 ед. при абсолютной децентрализации. Показатель ИДЦ представлял собой сумму величин спектральных гармоник матрицы множества функциональных состояний, которые обратно пропорциональны амплитуде спектральных гармоник и их дисперсии (подвижности). Были подобраны три группы пациентов одного возрастного диапазона от 40 до 65 лет (рис. 5):

группа 1 - контрольная, в которую вошли практически здоровые и больные с различными стадиями воспалительного процесса - альтерацией, воспалением, регенерацией. Количество 300 человек. ИДЦ $=45 \pm 30$ ед. (Mean $\pm \mathrm{SE})$ при $\mathrm{p} \leq 0.05$;

группа 2 - группа риска с доброкачественными опухолями и другими заболеваниями репаративной системы - гипертрофией простаты, миомой матки, полипозом кишечника, неспецифическим язвенным колитом, синдромом раздраженной толстой кишки, внутренним и наружным эндометриозом, болезнью Крона и по-

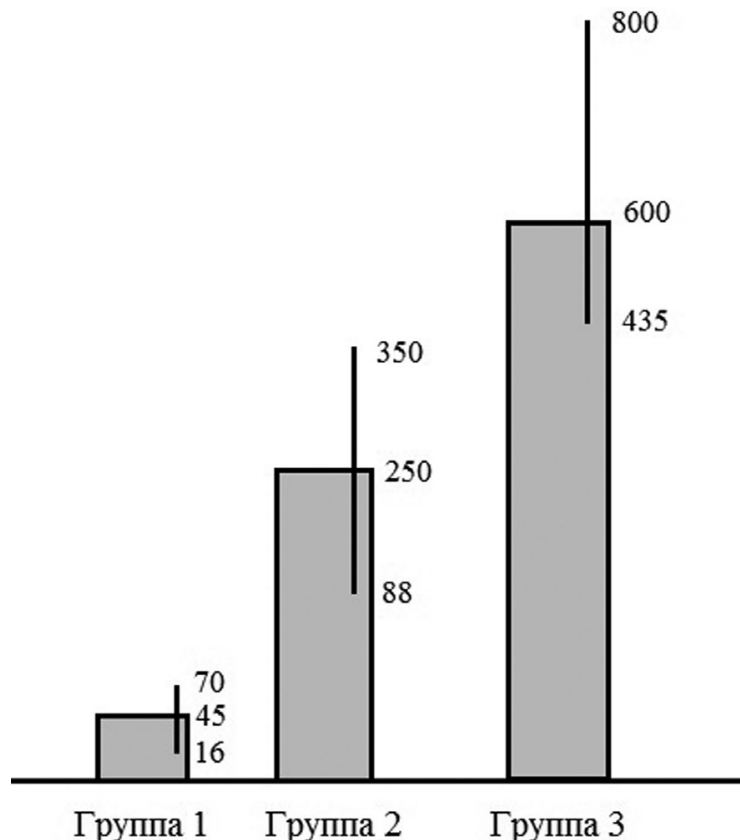

Puc. 5. Столбчатая диаграмма распределения индекса децентрализации (ИДЦ) по трем группам испытуемых. Группа 1 - не рак; группа 2 - доброкачественные пролиферативные заболевания; группа 3 гистологически подтвержденный рак. В каждой группе указаны среднее значение и стандартная ошибка Mean $\pm \mathrm{SE}$ при $\mathrm{p} \leq 0.05$

Fig. 5. Bar graph of the distribution of decentralization index of DCI (ИДЦ) through the three groups of subjects. Group 1 is no cancer; Group 2 is benign proliferative diseases; Group 3 is histologically confirmed cancer. In each group, the Mean $\pm \mathrm{SE}$ is indicated $(\mathrm{p} \leq 0.05)$

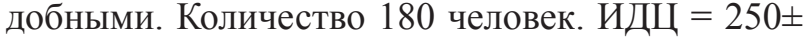
100 ед. (Mean $\pm \mathrm{SE})$ при $\mathrm{p} \leq 0.05$;

группа 3 - гистологически подтвержденная раковая опухоль кишечника, желудка, легких, головного мозга, печени, поджелудочной железы, предстательной железы, грудной железы и матки, надпочечников и почек. Количество 150 человек.

Группа 3 с гистологически подтвержденной раковой опухолью достоверно отличается от групп 1 и 2. У отдельных пациентов индекс децентрализации доходил до 1000 ед., что было связано со стадией и степенью распространенности раковой опухоли. ИДЦ $=600 \pm 100$ ед. (Mean \pm SE) при $\mathrm{p} \leq 0.05$.

В этом исследовании было достоверно показано, что со специфичностью $\mathrm{p}=0.8-0.9$ все случаи злокачественной эпителиальной опухоли отличаются от практически здоровых лиц по индексу ИДЦ. Причиной инициализации опухоли являлась децентрализация ткани со стороны центральной нервной системы. Как результат, можно предположить, что множественные очаги разрушения митохондрий и энергетического голодания клеток могут одновременно локализоваться в одном органе или образовывать «раковое поле» с захватом соседних органов. В наиболее выраженных очагах децентрализации запускаются механизмы мутации генетического аппарата клетки с целью избежать ее гибели (апаптоз клеток и некроз ткани). При этом происходит переход на анаэробный тип питания, неконтролируемое клеточное деление, потеря тканевой специфичности.

\section{ВЫВОДЫ}

Принципиально новый диагностический аппарат - «Регистратор спектра акустоэнцефалограммы РС АЭГ-01» позволяет за 3 мин регистрации информации получить многочастотную матрицу «множество функциональных состояний» висцерального анализатора и функциональный рельеф множества вегетативных функций организма. Большое количество спектральных гармоник - 8400, системное представление информации, высокая специфичность дают возможность назвать такую регуляторноанализаторную волновую структуру «висцером».

Анализ «матрицы множества функциональных состояний» позволил выделить ряд индексов для интегральной оценки состояния организма. На первом этапе отработаны:

ВИ - вегетативный индекс, ИН - индекс напряжения, ИИЗ - индекс индивидуального здоровья, ИДЦ - индекс децентрализации. На примере поведения во времени вегетативного индек- 
са ВИ в работе показана высокая корреляция его со стресс-индексом SI, который был получен из анализа КИГ в синхронных исследованиях. По данным АЭГ в разработке находятся индексы оценки глюкозы в крови, оценки иммунного статуса организма, решающие правила для диагностики ряда социально значимых патологических состояний. Это позволяет в сложных условиях эксплуатации внедрить оптимальную для таких условий индексную форму оценки состояния и мониторинга индивидуального здоровья индивида, создать сеть полностью автоматизированных диагностических пунктов, которые будут замыкаться по каналам связи на центральный сервер.

Аппарат РС АЭГ-01 обладает высокой помехозащищенностью, простотой в эксплуатации, неинвазивностью, скоростью регистрации и обработки информации, автономностью. Спектральный «висцером» содержит безграничную информацию о состоянии вегетативных функций, которая до конца не раскрыта. Волновое представление регуляторных звеньев организма должно существенно дополнить доминирующую в настоящее время морфофункциональную биохимическую модель, придать ей свойство организменной целостности.

\section{ЛИТЕРАТУРА}

Баевский Р. М. Оценка адаптационных возможностей организма и риск развития заболеваний. М. : Медицина, 1997. $236 \mathrm{c.}$

Баевский Р. М. Прогнозирование состояний на грани нормы и патологии. М. : Медицина, 1979. 295 с.

Булатеикий С. В. Соотношение уровня энергометаболических процессов и нейрогуморальной регуляции в управлении ритмом сердца // Актуальные вопросы общей патологии : в 2 т. / под ред. Ю. Ю. Белявского. Рязань : Изд-во РязГМУ, 2002. T. 1. C. 21-23.

Гнездиикий В. В. Обратная задача ЭЭГ и клиническая электроэнцефалография. М. : МЕДпрессинформ, 2004. $624 \mathrm{c}$.

Данилова Н. Н., Крылова А. Л. Физиология высшей нервной деятельности. Сер. «Учебники и учебные пособия». Ростов-на-Дону : Феникс, 2002. $480 \mathrm{c}$.

Жирмунская Е. А. В поисках объяснения феномена ЭЭГ. М. : НПФ Биола, 1995. 117 с.

Зимкина А. М. О критериях устойчивости в проявлениях деятельности центральной нервной системы человека // Физиол. журн. СССР. 1972. Т. 98, № 7. C. 1011-1018.

Кирой В. Н. Электроэнцефалография. Ростов-наДону : Изд-во РГУ, 1998. 239 с.

Мулик А. Б., Постнова М. В., Мулик Ю. А. Уровень общей неспецифической реактивности организма человека. Волгоград : Волгоград. науч. изд-во,
2009. $224 \mathrm{c}$.

Патент на полезную модель № 180056 Российская Федерация, МПК ${ }^{51}$ А61В 7/00. Регистратор спектра ритмической активности головного мозга / Шабанов Г. А., Лебедев Ю. А., Рыбченко А. А., Фейгин С. А., Зубков И. А. / НИЦ «Арктика» ДВО РАН (RU), заявка № 2017119102; приоритет 31.05.2017; опубл. 31.05.2018. Бюл. № 16.

Патент № 2661098 Российская Федерация, МПК ${ }^{51}$ А61B 5/05. Способ экспресс-диагностики онкологического заболевания / Рыбченко А. А., Шабанов Г. А., Максимов А. Л., Ищенко В. Н., Крыжановский С. П. / НИЦ «Арктика» ДВО РАН (RU), ГБОУ ВПО ТГМУ Минздрава России (RU); МО ДВО РАН (RU), заявка № 2016127302; заявл. 06.07.2016; опубл. 11.07.2018. Бюл. № 20.

Подольский И. Я., Воробьев В. В., Белова Н. А. Длительные изменения спектров ЭЭГ гиппокампа и неокортекса при фармакологических воздействиях на холинергическую систему // Ж. В. Н. Д. 2000. Т. 50. Вып. 6. С. 982-990.

Рольщиков А. И. Клинико-функциональная оценка метода компьютерной дермографии в диагностике, хирургическом лечении ранних и бессимптомных форм рака желудка : автореф. дис. ... канд. мед. наук. Владивосток, 2002.

Семенов Ю. Н. Комплекс для обработки кардиоинтервалограмм и анализа вариабельности сердечного ритма «Варикард 2.51»: руководство по эксплуатации ЮИМН.2.893.000 РЭ. 2010а. 296 с.

Семенов Ю. Н. Программное обеспечение Варикард-экспресс. ООО Институт Внедрения Новых Медицинских Технологий «РАМЕНА», $2010 б$. $44 \mathrm{c}$.

Хамошин А. B. Диагностика воспалительных и опухолевых заболеваний толстой кишки с использованием метода компьютерной дермографии : автореф. дис. ... канд. мед. наук. Владивосток, 1998.

Шабанов Г. А., Лебедев Ю. А., Рыбченко А. А., Maksimov, A. L.; Короченщев B. И. Исследование спектра акустического поля головного мозга человека // Вестник СВНЦ ДВО РАН. 2017. № 3. С. 115121.

Шабанов Г. А., Рыбченко А. А., Максимов А. Л. Модель активирующей системы пространственной организации биопотенциалов головного мозга: теоретическое и экспериментальное обоснование // Там же. 2005. № 1. C. 49-56.

Шабанов Г. А., Максимов А. Л., Рыбченко А. А. Функционально-топическая диагностика организма человека на основе анализа ритмической активности головного мозга. Владивосток : Дальнаука, 2011. $206 \mathrm{c}$.

Юдин С. В. Ранняя диагностика хирургических пролиферативных заболеваний с помощью современных компьютерных технологий : дис. ... канд. мед. наук. Владивосток, 1999.

Conny M. A. Heart rate variability // Annals of internal

Поступила в редакиию 01.11.2018 г.

Поступила после доработки 25.03.2019 2. 


\title{
NEW CLASS OF MEDICAL DIAGNOSTIC COMPLEXES BASED ON SPECTRAL ANALYSIS OF THE HUMAN BRAIN BIOACOUSTIC ACTIVITY
}

\author{
E. V. Pegova, G. A. Merkulova, Yu. A. Lebedev, G. A. Shabanov, A. A. Rybchenko \\ Scientific-Research Center "Arktika”, FEB RAS, Vladivostok
}

\begin{abstract}
The article discusses the development of objective instrumental methods for diagnosing functional states of an organism for extreme natural human habitats. A new diagnostic device for recording the spectrum of the brain bioacoustic activity has been offered. The spectrum of captured signals of head vibrations in the frequency range from 0.13 to $27 \mathrm{~Hz}$ is presented in the form of a multifrequency matrix of a set of functional states of a visceral analyzer, "viscerome." The quantity of spectral harmonics is 8400. A number of integral indicators have been developed for the automatic assessment of the obtained relief of the set of vegetative functions: vegetative index, stress index, individual health index, decentralization index. Exemplified by the vegetative index registration, functional tests are described: step test, return breathing test. The device permits to assesst the functional state and adaptive capacity of the organism, functional topical diagnosis of diseases, non-invasive screening of early forms of socially significant diseases, including oncology. The discussed technology permits to create a network of fully automated diagnostic points for monitoring individual health of the population. Connected to the central information processing server via communication channels such network would be vital for the complex operating conditions of the North and the North-East of Russia.
\end{abstract}

Keywords: acoustic encephalogram, spectrum registration, non-invasive screening, functional state, multi-frequency matrix, relief of visceral functions.

\section{REFERENCES}

Bayevsky, R. M., 1979, Prediction of Conditions on the Verge of Normal and Pathology, Moscow, Medicine [In Russian].

Bayevsky, R. M.; Berseneva A. P., 1997, Assessment of the Organism Adaptation Capabilities and the Risk of Developing Diseases, Moscow, Medicine [In Russian].

Bulatetsky, S. V., 2002, The Ratio of the Energy and Metabolic Processes Level and Neurohumoral Regulation in the Management of the Heart Rhythm, Current Issues of General Pathology, in 2 Vol., Ed. Yu. Yu. Belyavsky, Ryazan, Ryazan State Medical University, Vol. 1, 21-23 [In Russian].

Conny, M. A., 1993, Heart Rate Variability, Annals of Internal Medicine, 118, 436-447.

Danilova, N. N.; Krylova, A. L., 2002, Physiology of Higher Nervous Activity, Series "Textbooks and tutorials", Rostov-on-Don, Phoenix [In Russian].

Gnezditsky, V. V., 2004, Inverse Problem of EEG and Clinical Electroencephalography, Moscow, MEDpressinform [In Russian].

Khamoshin, A. V., 1998, Diagnosing Inflammatory and Neoplastic Diseases of the Colon Using the Computer Dermography Method, Diss... Cand. Sci. (Medicine), Vladivostok [In Russian].

Kiroy, V. N., 1998, Electroencephalography, Rostovon-Don, Rostov State University [In Russian].

Mulik, A. B.; Postnova, M. V.; Mulik, Yu. A., 2009, Level of General Non-Specific Reactivity of the Human Body, Volgograd, Volgogradskoye Nauchnoye Izd-vo [In Russian].
Podolsky, I. Ya.; Vorobyov, V. V.; Belova, N. A., 2000, Long-Term Changes in the EEG Spectra of the Hippocampus and Neocortex during Pharmacological Effects on the Cholinergic System, I. P. Pavlov Journal of Higher Nervous Activity, 50, 6, 982-990 [In Russian].

Rol'schikov, A. I., 2002, Clinical and Functional Assessment of the Computer Dermography Method in the Diagnosis and Surgical Treatment of Early and Asymptomatic Forms of Gastric Cancer, Diss. ... Cand. Sci. (Medicine), Vladivostok [In Russian].

Rybchenko, A. A.; Shabanov, G. A.; Maksimov, A. L.; Ищенко, В. Н.; Крыжжановский С. П., 2018, Method for Expressing Diagnostics of Oncological Disease, Patent No. 2661098, Russian Federation, MПK ${ }^{51}$ A61B 5/05, Bull. 20 [In Russian].

Semyonov, Yu. N., 2010a, "Varicard 2.51" Complex for Cardiointervalogram Processing and Analysis of Heart Rate Variability, Operation Manual, UIMN.2.893.000 RE [In Russian].

Semyonov, Yu. N., 2010b, Varicard-Express Software, Institute of Introduction of New Medical Technologies "RAMENA" [In Russian].

Shabanov, G. A.; Lebedev, Yu. A.; Rybchenko, A. A.; Feyghin, S. A; Zubkov, I. A., 2018, Recorder of Spectrum of Rhythmic Activity of Human Brain, Patent for Utility Model No. 180056, Russian Federation, МПК ${ }^{51}$ А61B 7/00, Bull. 16 [In Russian].

Shabanov, G. A.; Lebedev, Yu. A.; Rybchenko, A. A.; Maksimov, A. L.; Korochentsev, V. I., 2017, Investigation of the Spectrum of the Human Brain Acous- 
tic Field, Vestnik NESC FEB RAS, 3, 115-121 [In Russian].

Shabanov, G. A.; Maksimov, A. L.; Rybchenko, A. A., 2011, Functional-Topical Diagnosis of the Human Body Based on an Analysis of the Brain Rhythmic Activity, Vladivostok, Dalnauka [In Russian].

Shabanov, G. A.; Rybchenko, A. A.; Maksimov, A. L., 2005, Model of the Activating System of the Spatial Organization of Brain Potentials: Theoretical and Experimental Grounds, Vestnik NESC FEB RAS, 1, 49-56 [In Russian].
Yudin, S. V., 1999, Early Diagnosis of Surgical Proliferative Diseases with the Help of Modern Computer Technologies, Diss. ... Cand. Sci. (Medicine), Vladivostok [In Russian].

Zhirmunskaya, E. A., 1995, In Search for an Explanation of the EEG Phenomenon, Moscow, Biola [In Russian].

Zimkina, A. M., 1972, On Stability Criteria in Manifestations of the Activity of Human Central Nervous System, Fiziologichesky Zhurnal SSSR, 98, 7, 1011-1018 [In Russian]. 